\title{
Prospective Respiratory Gated Carbon Nanotube Micro Computed Tomography
}

\author{
Yueh Z. Lee, MD, PhD ${ }^{1,2}$, Laurel M. Burk, BS ${ }^{2}$, Ko-han Wang, BS ${ }^{1}$, Guohua Cao, PhD², \\ Jonathan Volmer, $\mathbf{P h D}^{5}$, Jianping $\mathbf{L u}, \mathbf{P h D}^{2}$, and Otto Zhou, $\mathbf{P h D}^{2,3,4}$ \\ ${ }^{1}$ Department of Radiology, University of North Carolina, Chapel Hill, NC 27599 \\ 2Department of Physics and Astronomy, University of North Carolina, Chapel Hill, NC 27599 \\ ${ }^{3}$ Curriculum in Applied Sciences and Engineering, University of North Carolina, Chapel Hill, NC \\ 27599 \\ ${ }^{4}$ Lineberger Comprehensive Cancer Center, University of North Carolina, Chapel Hill, NC 27599 \\ ${ }^{5}$ Cystic Fibrosis Center, University of North Carolina, Chapel Hill, NC 27599
}

\begin{abstract}
Objectives-Challenges remain in the imaging of the lungs of free-breathing mice. Though computed tomography (CT) is near optimal from a contrast perspective, the rapid respiration rate, limited temporal resolution and inflexible x-ray pulse control of most micro-CT (mCT) scanners limits their utility in pulmonary imaging. Carbon nanotubes (CNTs) have permitted the development of field emission cathodes, with rapid switching and precise pulse control. The goal of this study was to explore the utility of a CNT-based mCT for application in quantitative pulmonary imaging.
\end{abstract}

Materials and Methods-Twelve CB57/B6 mice were imaged during peak-inspiration and end-exhalation using the CNT mCT system. The respiratory trace was derived from a sensor placed underneath the abdomen of the animal. Animals were allowed to free-breathe during the imaging under isoflurane anesthesia. Images were reconstructed using to isotropic voxels of 77 micron resolution (50 kvP, 400 projections, $30 \mathrm{msec} x$-ray pulse). Lung volumes were measured with region growing techniques and thresholds derived from the surrounding air and soft tissues. Basic functional parameters including tidal volume, functional reserve capacity and minute volume were also calculated.

Results-Average scan time was $13.4+/-1.8$ minutes for each phase of the respiratory cycle. Mean lung volumes at peak inspiration and end-expiration were $0.23+/-0.026$ and $0.11+/-0.024$ $\mathrm{ml}$, respectively. The average minute volume was $11.93+/-2.64 \mathrm{ml} / \mathrm{min}$.

Conclusion-We demonstrate the utility of a CNT based micro-CT in acquiring prospectively gated images from free breathing mice for obtaining physiological data. This technique provides an alternative to breath-hold techniques requiring intubation and offers a more dose efficiency than retrospective gating techniques.

(C) 2011 The Association of University Radiologists. Published by Elsevier Inc. All rights reserved.

Corresponding Author \& Request for reprints: Yueh Z. Lee, The University of North Carolina at Chapel Hill, Phillips Hall, CB \#3255, Chapel Hill, NC 27599-3255, yueh@alum.mit.edu, Fax: 919-962-0480, Phone: 919-423-7195.

Publisher's Disclaimer: This is a PDF file of an unedited manuscript that has been accepted for publication. As a service to our customers we are providing this early version of the manuscript. The manuscript will undergo copyediting, typesetting, and review of the resulting proof before it is published in its final citable form. Please note that during the production process errors may be discovered which could affect the content, and all legal disclaimers that apply to the journal pertain. 


\section{INTRODUCTION}

The lung continues to be a challenging organ for evaluation with high-resolution microcomputed tomography (micro-CT) imaging. The large difference in attenuation between air and soft tissue results in an optimal contrast environment for CT based techniques, with great potential to be useful in a number of applications, including toxicology and oncology. 1-3 However, the rapid respiratory rate of the most important biomedical model, the mouse, limits evaluation with standard micro-CT systems. In contrast, organ systems with limited associated motion at rest, such as the extremities, have had significantly more micro-CT imaging applications demonstrated.4

The respiratory rate of mice ranges from 120 to 300 breaths per minute (BPM), resulting in a maximum breath to breath period of approximately $500 \mathrm{msec}$. To "freeze" the motion a single projection image needs to be captured with an equivalent exposure of approximately $100 \mathrm{msec}$ or less. One approach is to intubate the animal and provide full control of the animal's respiration. This approach allows complete control of the animal's respiration cycle, allowing imaging at almost any point within the cycle.5 Practically, however, intubation is associated with airway trauma and by removing the underlying physiological drive to breathe, obtaining true physiological parameters using this technique would be difficult. Obtaining these physiological parameters, however, is of great research interest. 6 Thus, from a pulmonary physiology perspective the preference is to perform non-intubated imaging.

Two major methods may be applied to perform non-intubated respiratory gated micro-CT imaging of murine models. The first, retrospective gating, acquires a respiratory trace, typically using a pressure sensor during repeated, rapid imaging of the animal. Multiple complete sets of CT projections are obtained. The respiratory trace corresponding to each projection is analyzed to identify the projections that occur within the phase of the respiratory cycle of interest. These projections are then assembled together and reconstructed. Though relatively time efficient, this method can result in a large amount of wasted dose. In contrast, the second technique, prospective respiratory gating, offers significant dose efficiency by firing an x-ray pulse only when the respiratory cycle has reached a specific point. Sufficient data for a full CT reconstruction may then be obtained by acquiring additional projections at the same portion of subsequent respiratory cycles.

We have previously demonstrated that a field emission, carbon nanotube (CNT) based x-ray source can produce $\mathrm{x}$-ray pulses of sufficient flux for small animal imaging on demand.7 The goal of this study is to explore the application of this system for quantitative evaluation of lung function of normal mice.

\section{MATERIALS AND METHODS}

A custom built micro-CT scanner based on a carbon nanotube cathode and flat-panel detector was utilized for the imaging studies. 8 The $\mathrm{x}$-ray source consists of a carbon nanotube cathode with a tungsten target. A cesium iodine based flat panel detector, C7940DK, (Hamamatsu Corp, Hamamatsu Japan) detector with $12 \times 12 \mathrm{~cm}$ field of view, 50 micron pixel size was positioned opposite the $x$-ray source on a rotation gantry. Only the central $5 \times 5 \mathrm{~cm}$ area of the detector was utilized for imaging. The approximate field of view of the setup was $4.0 \mathrm{~cm}$ in diameter by $5 \mathrm{~cm}$ in length. The overall scanner resolution was 77 $\mu \mathrm{m}$ in this configuration. A custom animal bed was designed and printed in acrylonitrile butadiene styrene (ABS) plastic.

A standard imaging protocol was utilized with 400 projections over a 200 degree field of view with a step-and-shoot protocol. Each X-ray pulse was 30 milliseconds in duration at 50 
$\mathrm{keV}$ energy. The system control software was written in Labview (National Instruments Corp, Austin TX) and provided synchronization of the x-ray detector and X-ray source with triggering from the physiological monitoring. Minimum scan time of the system is 400 seconds to allow for gantry rotation and image readout. Physiological gating was acquired using a Biovet monitor (m2m Imaging Corp, Cleveland $\mathrm{OH}$ ); the respiration sensor was secured underneath the abdomen of the animal on the custom bed. Hounsfield unit correction was then performed based on known correction factors for the system using a water bath and air.

\section{Image acquisition}

All studies were performed under an approved Institutional Animal Care and Use Committee protocol. A total of twelve wild-type C57/Black6J strain female mice were imaged using the system. The animals were anesthetized initially using $3.0 \%$ isoflurane with a 1.5\% maintenance dose delivered through a nose cone integrated into the custom animal bed. The isoflurane dose was modulated to maintain the animal respiration rates between 80 to 120 breaths per minute. After placing the animals onto the bed, they were secured with a small piece of non-adhesive stretchable bandage, Coban (3M Medical, St. Paul, MN)

Two sets of CT images were obtained for each animal, at peak inspiration and end expiration. Thresholds levels were set within the Biovet software to the peak of the respiration cycle pressure, corresponding to peak inspiration. A variable $150-200 \mathrm{msec}$ delay from the peak inspiration point enabled identification of the end expiration point. The delay and threshold selection proved fairly consistent throughout the respiratory cycle, even with mild variations of the respiration rate.

\section{Image Reconstruction and Analysis}

Cone beam CNT x-ray source CT reconstructions were performed with Cobra (Exxim Corporation, Pleasanton, CA). Preprocessing was performed with custom software written in Matlab (Mathworks Inc, Natick MA) to eliminate bad pixels and correct for cone beam heterogeneity. Total reconstruction time including preprocessing was performed in approximately 3 minutes per data set. Data were reconstructed into DICOM compatible formats to simplify post-processing and data storage.

Post-processing was performed with OsiriX imaging software. 9 Region growing analysis was utilized to calculate lung volumes using thresholds established for each image. Two thresholds were established, the first separating soft tissue from lung parenchyma, and the second separating lung from the major airways. The lung and trachea volume within the thoracic cavity was first segmented using multiple seed points in the trachea and lung. Trachea volume was then independently measured using seed points positioned within only the trachea with region growing occurring in a cranial to caudal direction. The voxel volume of these two components could then be calculated. Lung density was also measured in Hounsfield units and used to correct the voxel volumes of the lungs to the actual air volume. Tidal lung volume could then be measured with simple subtraction. Other potential parameters of interest, including trachea diameter, were directly measured using OxiriX.

\section{RESULTS}

A total of 12 normal, wild-type mice were imaged. Average scan time was $13.4+/-1.8$ minutes for each phase of the respiratory cycle. Average respiration rate was $96.2+/-7.1$ breaths per minute for the mice.

Lung volumes were measured for each animal using a region growing technique within the OsiriX software. Thresholds were derived for each animal based on the average soft tissue 
attenuation value as the upper level threshold. A few (three to five) initial seed points were placed in the trachea and the left and right lobes of the lungs. The region growing extended to fill both the lungs and trachea. A second, lower bound threshold was derived for the lung parenchyma; a region growing volume initiated with seed point in the upper trachea defines the major airways. The difference between these two volumes for each phase of the respiration cycle represents the uncorrected total lung volume. The lung volume is then corrected for density by incorporating the density (measured in HU) of the lung parenchyma using Equation 1 for both inspiration and expiration states. Tidal volume may then be calculated as the difference between the two phases of the respiratory cycle, end-expiration and peak inspiration. Table 1 contains the total lung volume measurements for each phase of the respiratory cycle, tidal volume, and tracheal diameter. Derived functional pulmonary parameters are listed in Table 2 with parameters from a recent respiratory gated lung imaging study. 10 Functional reserve capacity (FRC) is the lung volume at end expiration. Tidal Volume is the difference between peak inspiration and end-expiration (Equation 2). Minute Volume is the product of tidal volume and the respiration rate and represents the total amount of gas respired by the animal in one minute. These parameters represent the basic lung physiological parameters.

$$
\overline{\text { Air }} \text { Density(HU) Equation 1: }
$$

$$
\text { Tidal Volume }(\mathrm{ml})=\text { Lung } \text { Volume }_{\text {expiration }}-\text { Lung Volume } \text { inspiration }_{\text {Equation 2: }}
$$

Sample images of a single mouse lung in full-expiration and peak-inspiration are seen in Figure 1, with axial and coronal reformatted coronal images. Rendered images of the lungs and trachea in peak inspiration and end-expiration are shown in Figure 2. Differences in rib spacing are seen that demonstrate the changes in the phase of the respiratory cycle.

\section{DISCUSSION}

In this study, we demonstrate the utility of a carbon nanotube based micro-CT system in the acquisition of functional pulmonary parameters in mice that are anesthetized, but not intubated. Figure 1 demonstrates the surface shaded renderings of the lungs that demonstrate the qualitative differences between peak inspiration and end-expiration of a sample set of mouse lungs. Changes in the rib spacing, and cranial-caudal dimension of the lungs can be seen in the renderings.

We note that our derived anatomic parameters are similar to a recent study by Ford et. al, with slight differences in the tidal volume of the animal. Our derived tidal volume is approximately $30 \%$ greater than the other study. However, when one takes into account our decreased respiration rate, presumably due to a higher level of anesthesia, the difference in minute volume does not appear to be significant. While this equivalency could be coincidental, we feel that it is reflective of measuring the underlying physiology appropriately as the minute volume needs of a sedated animal should not be dramatically different, as the basal oxygen requirements and respiratory drive should be equivalent.

One distinct advantage of our CNT based sources is the ability to generate an x-ray pulse of any duration. Based on our experience with the respiratory traces, we have found that 50 msec or shorter x-ray pulses are sufficient to capture the respiratory motion; we arbitrarily chose $30 \mathrm{msec}$ to minimize any respiratory motion. Given the upper limits of respiration rate in our study, of approximately $500 \mathrm{sec}$, the $30 \mathrm{msec}$ would represent only $6 \%$ of the respiratory cycle. In contrast, other systems are limited by the system x-ray shutter resolution available, such as $170 \mathrm{msec}$ in other studies10. More recently, one group has 
utilized a single-view approach to estimating lung volumes using a $10 \mathrm{msec} x$-ray generator. 11 Though the CNT source could be trivially adapted to this technique, the estimation of a three-dimensional volume through a single planar projection is of limited utility and likely only useful in specific, well-calibrated applications.

A number of groups have bypassed the extreme needs of mouse imaging by using the rat as an alternate model of interest. 12-14 Since the rat is roughly five to ten times the size of the mouse, with comparable reductions in respiration rate and appropriate increases in lung size, the image requirements would be less strenuously. Unfortunately, the reduced genetic model variability and the larger animal size also limits the overall utility of these models.

A few approaches to gated lung micro-CT imaging have been demonstrated in the literature. These approaches can be divided into two major categories; using non-invasive and invasive gating techniques. The invasive approaches require intubation of the animal to provide full control of the respiration of the animal, including the use of a small animal respirator and need for a neuromuscular blocker pharmaceutical.

A number of approaches to intubation are described in the literature, however, the invasive procedure of placing a rigid or semi-rigid tube into the trachea risks injury to the trachea and structures in the vicinity. 15 Furthermore, there is likely ventilation induced injury even at the least injurious mechanical ventilator settings. 16,17 Eliminating the potential for injury would reduce the number of experimental animals needed for a study and reduce confounding factors. The animal's natural respiratory drive is also eliminated when the required paralyzing agent is administered, and free-breathing respiratory parameters cannot be measured. For researchers interested in lung physiology, this is of great importance as other approaches to obtain these useful parameters of interest may not be available. Intubation of the animal does confer significant advantages in synchronization, especially for systems that are unable to fire x-ray pulses on demand. The lung volume may be maintained at a specific lung volume or pressure using a "breath-hold" technique. 18 However, there are clear advantages from not requiring intubation when the system is capable of synchronization of the x-ray sources, such as the flexibility offered by the CNT $\mathrm{x}$-ray sources. If intubation is desired or necessary within an imaging protocol, precise synchronization with the "on-demand" CNT sources is also simplified.

Others have approached the capture of lung motion by using retrospective gating techniques. This is implemented through a micro-CT system with flat-panel based detector and continuous image acquisition capability. The system obtains images of the region of interest throughout multiple phases of the respiratory cycle. Each projection can then be correlated with a specific phase of the respiratory cycle, and placed into the appropriate bin for reconstruction of each phase. Scan times with this technique are very short, since no gantry or exposure window synchronization are required. 19 However, there is also significant wasted x-ray dose, especially if only a limited number of phases of the respiratory cycle are required. For example, the end-expiration and full-inspiration phases represent approximately $20 \%$ of the entire respiratory cycle, from which many of the basic dynamic respiration parameters of interest can be derived. If a researcher is only interested in these two phases, then the remaining $80 \%$ of the scan will not be utilized, and is wasted dose. This excess dose estimate is under optimal conditions; significant oversampling of the same projections within the same phase may also occur, resulting in redundant projections that do not contribute to appropriate angular coverage. Given the potential genetic changes and the importance of longitudinal studies, a technique that minimizes radiation exposure remains a priority. Our imaging dose is $12.5 \mathrm{cGy}$, which is comparable to other prospectively gated systems allowing for differences in protocol and resolution. 3,20 In contrast to other systems 
however, our use of the CNT enabled x-ray sources allows for more precise control of the Xray source.

Furthermore, most commercially available flat panel detectors do not have sufficiently high readout rates to allow for the rapid data acquisition. Instead, custom detectors with pixel binning or custom readout amplifiers are necessary to maintain high acquisition rates, at the loss of resolution and increased expense. Thus, precise operational control of the CNT x-ray sources confers significant advantages in only activating the source when an exposure is required. Furthermore, if combined with a high-speed flat panel system, the CNT x-ray source could allow similar reductions in scan time without additional dose.

As others have reported, we found the quality of the respiration signal to be dependent on the tension of the band securing the animal to the respiratory sensor. Excessive pressure causes atelectasis (reversible collapse of the portions of the lung spaces) and will cause erroneous lung volume measurements. With appropriate operator training, the potential for this can be minimized; however, it is clear that if non-contact or non-compressive approaches to identifying the gating signal can be established, this effect could be eliminated. We are currently exploring such techniques with our system.

FRC and $\mathrm{V}_{\mathrm{t}}$ represent the basic lung physiology parameters that may be derived using this technique. We recognize that the underlying physiology and regulatory mechanisms are likely altered by the use of an anesthetic agent. 21 However, there is no physiological reason that the system may not be utilized in a non-anesthetized animal, if appropriate gating and/or restraint systems can be employed. Different methods of anesthesia may also be selected to minimize their effects on the animal's respiratory drive. Furthermore, multiple phases of the respiratory cycle may also be obtained to produce the equivalent of a small animal forced expiratory volume, which may be a useful adjunct in pulmonary tests, especially for models interested in airway physiology. Additional phases of the respiratory cycle would require additional dose to the animal, however.

We demonstrate the utility of CNT based x-ray sources for the acquisition of gated respiratory images in free-breathing mice. Not requiring intubation of the animal also simplifies animal handling and setup, while reducing trauma to the animal itself. The flexibility of control of the CNT allows more precise control over the x-ray source, flux rates high enough for rapid imaging and sufficient system stability. One other distinct advantage of our CNT based system is that it can also be adapted to almost any other approach to lung imaging, but with better x-ray pulse control and system simplification. Overall, our flexible system allows biomedical researchers to obtain useful physiologic lung parameters to be measured from murine models.

\section{Acknowledgments}

Funding: National Institutes of Health-NIBIB (4R33EB004204-01), National Institutes of Health-NCI (U54CA119343) and Xintech Inc.

\section{REFERENCES}

1. Ford NL, Martin EL, Lewis JF, et al. Quantifying lung morphology with respiratory-gated micro-CT in a murine model of emphysema. Phys Med Biol. 2009; 54:2121-2130. [PubMed: 19287083]

2. Shofer S, Badea C, Qi Y, et al. A micro-CT analysis of murine lung recruitment in bleomycininduced lung injury. J. Appl. Physiol. 2008; 105:669-677. [PubMed: 18566189]

3. Cody DD, Nelson CL, Bradley WM, et al. Murine lung tumor measurement using respiratory-gated micro-computed tomography. Invest Radiol. 2005; 40:263-269. [PubMed: 15829823] 
4. Schambach SJ, Bag S, Schilling L, Groden C, Brockmann MA. Application of micro-CT in small animal imaging. Methods. 2010; 50:2-13. [PubMed: 19706326]

5. Walters EB, Panda K, Bankson JA, Brown E, Cody DD. Improved method of in vivo respiratorygated micro-CT imaging. Phys Med Biol. 2004; 49:4163-4172. [PubMed: 15470930]

6. O'Neil JJ, Raub JA. Pulmonary function testing in small laboratory mammals. Environ Health Perspect. 1984; 56:11-22. [PubMed: 6434299]

7. Cheng Y, Zhang J, Lee YZ, et al. Dynamic radiography using a carbon-nanotube-based fieldemission x-ray source. Rev. Sci. Instrum. 2004; 75:3264-3267.

8. Cao G, Lee YZ, Peng R, et al. A dynamic micro-CT scanner based on a carbon nanotube field emission x-ray source. Phys Med Biol. 2009; 54:2323-2340. [PubMed: 19321922]

9. Rosset A, Spadola L, Ratib O. OsiriX: An Open-Source Software for Navigating in Multidimensional DICOM Images. Journal of Digital Imaging. 2004; 17:205-216. [PubMed: 15534753]

10. Ford NL, Martin EL, Lewis JF, et al. In vivo characterization of lung morphology and function in anesthetized free-breathing mice using micro-computed tomography. J Appl Physiol. 2007; 102:2046-2055. [PubMed: 17255374]

11. Lai-Fook SJ, Houtz PK, Lai Y. End-expiratory and tidal volumes measured in conscious mice using single projection x-ray images. J Appl Physiol. 2008; 104:521-533. [PubMed: 17872404]

12. Jobse BN, Johnson JR, Farncombe TH, et al. Evaluation of allergic lung inflammation by computed tomography in a rat model in vivo. Eur. Respir. J. 2009; 33:1437-1447. [PubMed: 19164353]

13. Ford NL, Nikolov HN, Norley CJD, et al. Prospective respiratory-gated micro-CT of free breathing rodents. Med Phys. 2005; 32:2888-2898. [PubMed: 16266103]

14. Ask K, Labiris R, Farkas L, et al. Comparison between conventional and "clinical" assessment of experimental lung fibrosis. J Transl Med. 2008; 6:16. [PubMed: 18402687]

15. Brown RH, Walters DM, Greenberg RS, Mitzner W. A method of endotracheal intubation and pulmonary functional assessment for repeated studies in mice. J Appl Physiol. 1999; 87:23622365. [PubMed: 10601190]

16. Curley GF, Kevin LG, Laffey JG. Mechanical Ventilation. Anesthesiology. 2009; 111:701-703. [PubMed: 19707117]

17. Wolthuis E, Vlaar A, Choi G, et al. Mechanical ventilation using non-injurious ventilation settings causes lung injury in the absence of pre-existing lung injury in healthy mice. Critical Care. 2009; 13:R1. [PubMed: 19152704]

18. Namati E, Chon D, Thiesse J, et al. In vivo micro-CT lung imaging via a computer-controlled intermittent iso-pressure breath hold (IIBH) technique. Phys Med Biol. 2006; 51:6061-6075. [PubMed: 17110770]

19. Bartling SH, Stiller W, Grasruck M, et al. Retrospective motion gating in small animal CT of mice and rats. Invest Radiol. 2007; 42:704-714. [PubMed: 17984768]

20. Cavanaugh D, Johnson E, Price RE, et al. In vivo respiratory-gated micro-CT imaging in smallanimal oncology models. Mol Imaging. 2004; 3:55-62. [PubMed: 15142412]

21. Eger EI. The pharmacology of isoflurane. Br J Anaesth. 1984; 56(Suppl 1):71S-99S. [PubMed: 6391530] 


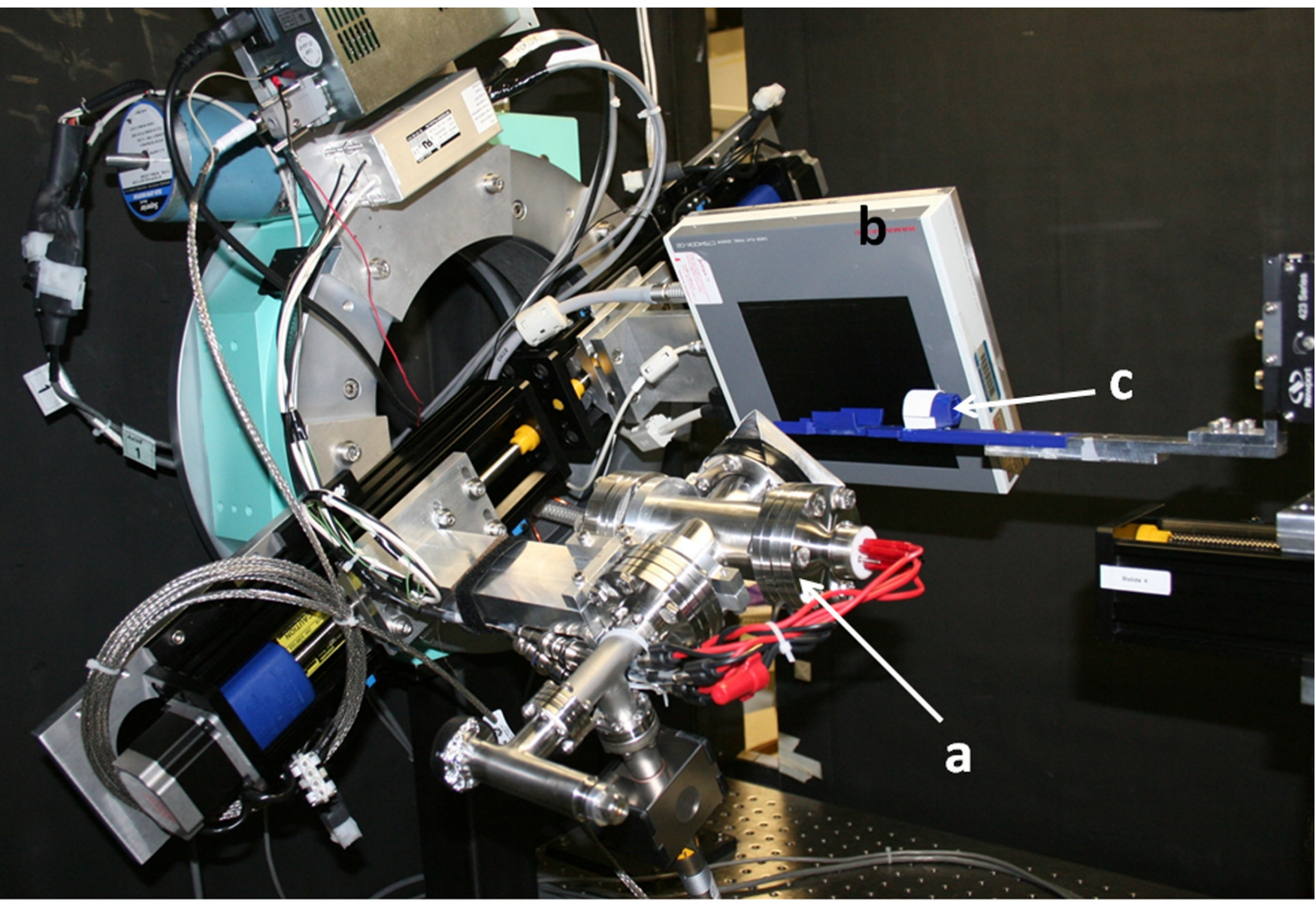

Figure 1.

Shaded surface renderings of a mouse lung and trachea in inspiration (a) and expiration (b). Changes in the shape and dimensions of the lungs can be appreciated. 

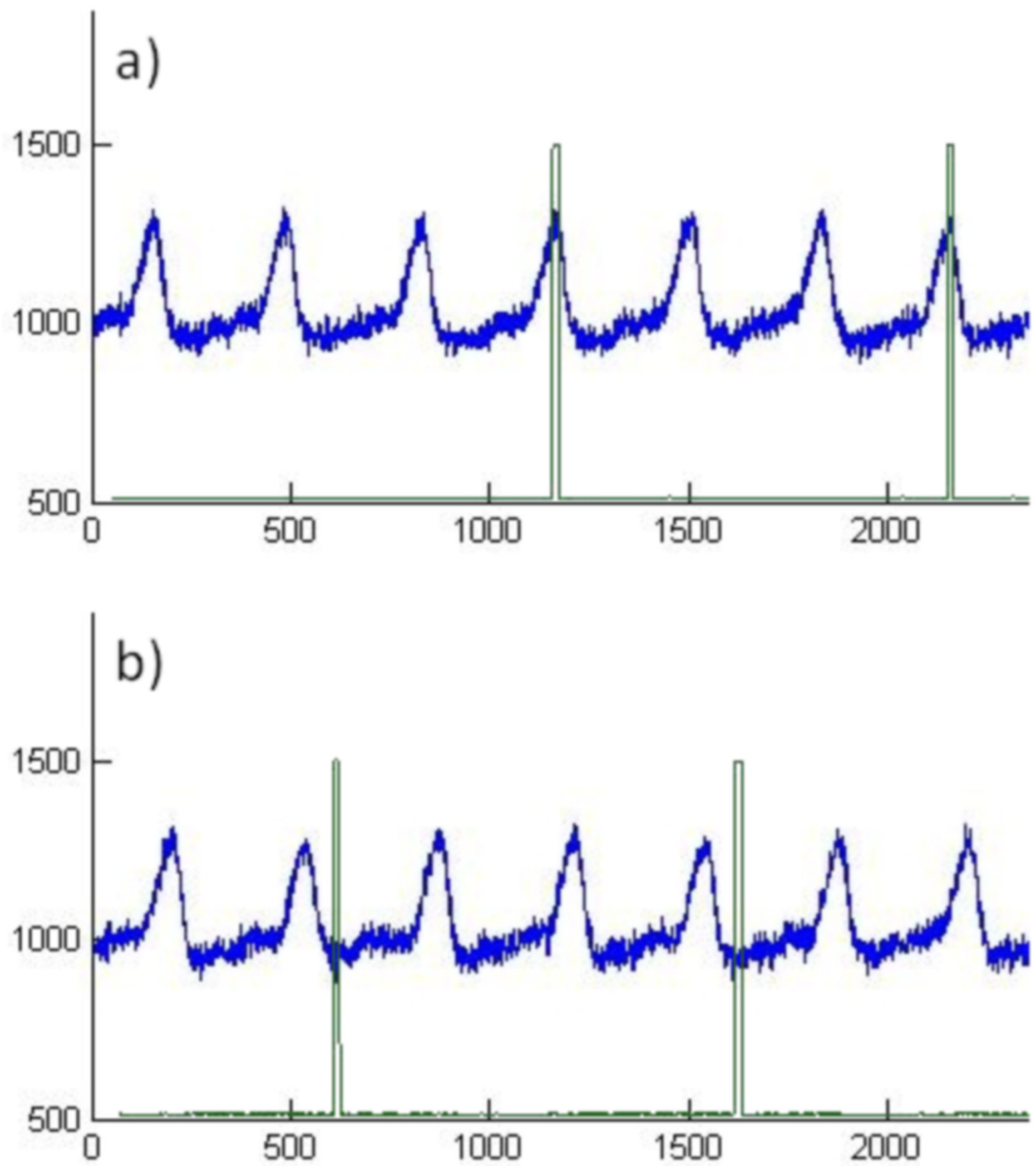

Figure 2.

Axial $(a, b)$ and reformatted coronal (c, d) images obtained at the same slice location obtained during peak-inspiration (a, c) and full-expiration(b, d) through the lower lung of a single animal. 


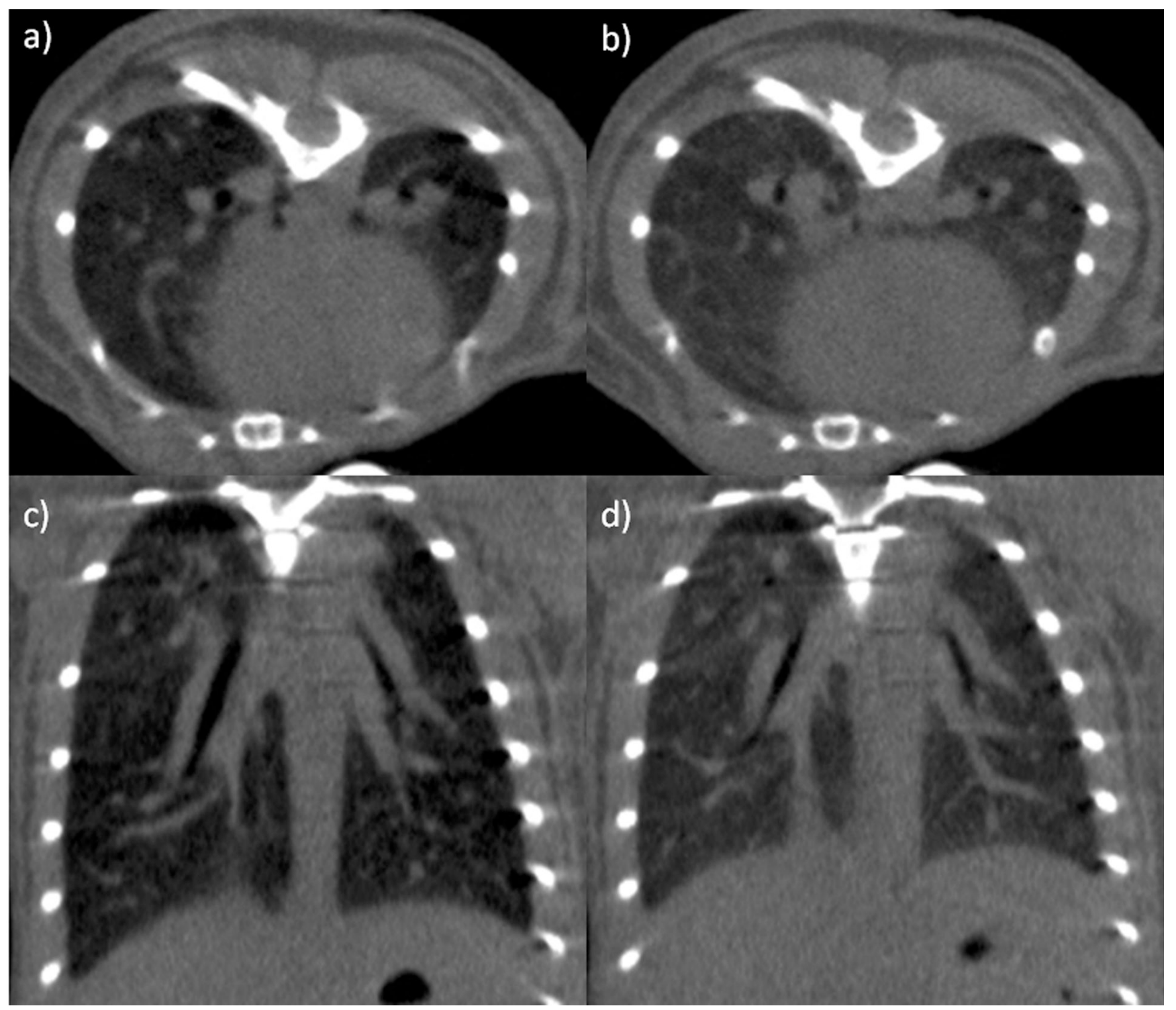

Figure 3.

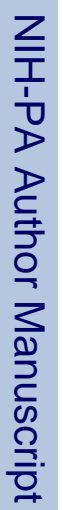




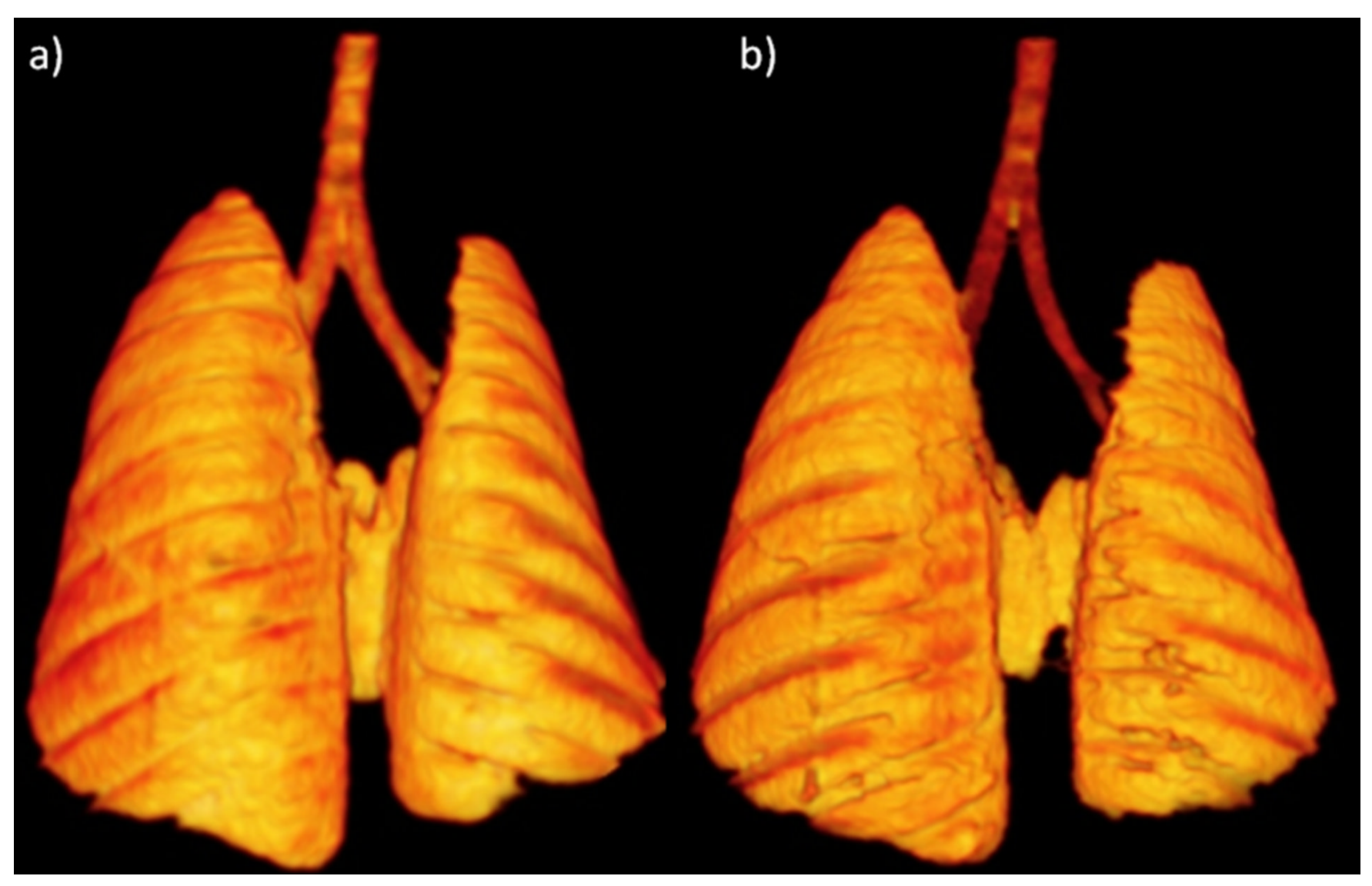

Figure 4. 


\section{Table 1}

Comparison of respiration rate, trachea diameter, organ and parenchymal volume at peak inspiration and end expiration for imaged mice.

\begin{tabular}{|l|c|c|c|c|}
\hline \multirow{2}{*}{} & \multicolumn{2}{|c|}{ Peak Inspiration } & \multicolumn{2}{c|}{ End Expiration } \\
\cline { 2 - 5 } & Mean & STD & Mean & STD \\
\hline Respiration Rate $(\mathrm{bpm})$ & 92 & 8.0 & 101 & 10.5 \\
\hline Trachea diameter $(\mathrm{mm})$ & 0.94 & 0.067 & 0.88 & 0.071 \\
\hline Organ Volume $(\mathrm{ml})$ & 0.41 & 0.071 & 0.26 & 0.069 \\
\hline Parenchymal Volume $(\mathrm{ml})$ & 0.23 & 0.026 & 0.11 & 0.024 \\
\hline
\end{tabular}




\section{Table 2}

Comparison of functional reserve capacity (FRC), tidal volume (TV) and minute volume between the current study and a recent study by Ford et. al (Reference 10).

\begin{tabular}{|l|c|c|c|c|}
\hline \multirow{2}{*}{} & \multicolumn{2}{|c|}{ Ford et. al } & \multicolumn{2}{c|}{ Current study } \\
\cline { 2 - 5 } & Mean & STD & Mean & STD \\
\hline Functional Reserve Capacity $(\mathrm{ml})$ & 0.16 & 0.03 & 0.11 & 0.02 \\
\hline Tidal Volume $(\mathrm{ml})$ & 0.09 & 0.03 & 0.12 & 0.02 \\
\hline Minute Volume (ml/min) & 12.47 (est.) & 4.2 (est.) & 11.93 & 2.64 \\
\hline
\end{tabular}

